\title{
Chromoanagenesis: cataclysms behind complex chromosomal rearrangements
}

Franck Pellestor ${ }^{1,2}$ (i)

\begin{abstract}
Background: During the last decade, genome sequencing projects in cancer genomes as well as in patients with congenital diseases and healthy individuals have led to the identification of new types of massive chromosomal rearrangements arising during single chaotic cellular events. These unanticipated catastrophic phenomenon are termed chromothripsis, chromoanasynthesis and chromoplexis., and are grouped under the name of "chromoanagenesis".

Results: For each process, several specific features have been described, allowing each phenomenon to be distinguished from each other and to understand its mechanism of formation and to better understand its aetiology. Thus, chromothripsis derives from chromosome shattering followed by the random restitching of chromosomal fragments with low copy-number change whereas chromoanasynthesis results from erroneous DNA replication of a chromosome through serial fork stalling and template switching with variable copy-number gains, and chromoplexy refers to the occurrence of multiple inter-and intra-chromosomal translocations and deletions with little or no copynumber alterations in prostate cancer. Cumulating data and experimental models have shown that chromothripsis and chromoanasynthesis may essentially result from lagging chromosome encapsulated in micronuclei or telomere attrition and end-to-end telomere fusion.

Conclusion: The concept of chromanagenesis has provided new insight into the aetiology of complex structural rearrangements, the connection between defective cell cycle progression and genomic instability, and the complexity of cancer evolution. Increasing reported chromoanagenesis events suggest that these chaotic mechanisms are probably much more frequent than anticipated.
\end{abstract}

Keywords: Chromoanagenesis, Chromothripsis, Chromoanasynthesis, Chromoplexy, Cancer, Evolution, Genome instability, Micronucleus, Double-strand breaks, Telomeres, Replication

\section{Background}

Over the past decade, genome sequencing effort combining new generation DNA sequencing technologies and efficient bioinformatics tools have lead to the discovery of new types of complex and massive chromosomal and genomic alterations characterized by the simultaneous occurrence of multiple structural rearrangements confined to one or a few chromosomal segments through a single catastrophic event. Grouped under the term of chromoanagenesis (for chromosome rebirth), this new class of genomic alterations involve 3 distinct phenomenons: the

\footnotetext{
Correspondence: fpellestor@yahoo.fr

${ }^{1}$ Unit of Chromosomal Genetics, Department of Medical Genetics, Arnaud de Villeneuve Hospital, Montpellier CHRU, 371, avenue du Doyen Gaston Giraud, 34295 Montpellier cedex 5, France

${ }^{2}$ INSERM 1183 Unit «Genome and Stem Cell Plasticity in Development and Aging », Institute of Regenerative Medicine and Biotherapies, St Eloi Hospital, Montpellier, France
}

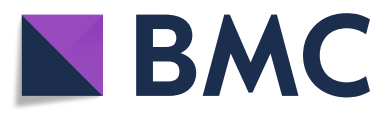

(c) The Author(s). 2019 Open Access This article is distributed under the terms of the Creative Commons Attribution 4.0 International License (http://creativecommons.org/licenses/by/4.0/), which permits unrestricted use, distribution, and reproduction in any medium, provided you give appropriate credit to the original author(s) and the source, provide a link to the Creative Commons license, and indicate if changes were made. The Creative Commons Public Domain Dedication waiver (http://creativecommons.org/publicdomain/zero/1.0/) applies to the data made available in this article, unless otherwise stated. plexy [1].

The concept of chromoanagenesis provides new insight into the nature of complex chromosomal rearrangements. Both the complexity and the diversity of chromoanagenesis-related rearrangements raise important questions concerning the cellular mechanisms driving chromoanagenesis events, the aetiology of these chaotic processes and their impact in human pathology. Experimental models allowed to validate the existence of these catastrophic phenomenon, and to evidence some of the causative mechanisms. In this review, are summarized exciting data and recent progress on understanding the formation and the consequences of these complex genomic alterations. 


\section{Chromothripsis}

Chromothripsis is the first of these new catastrophic process (mechanism) described in 2011 [2]. The phenomenon is currently defined as a mutational event driven by multiple double-strand breaks (DSBs) occurring in a single catastrophic event between a limited numbers of chromosomal segments, and followed by the reassembly of the DNA fragments in random order and orientation to form complex derivative chromosomes (Fig. 1).

Several factors common to all chromothripsis events, such as the generation of numerous clustered chromosomal breakpoints, the low DNA copy number changes and the preservation of heterozygosity in the rearranged segments, allow to distinguish chromothripsis from other complex chromosomal rearrangements and define its molecular signature $[3,4]$.

Initially described in cancers [1], the phenomenon was rapidly evidenced in patients with congenital abnormalities [5-7]. Notably, even some translocations and inversions classified as simple balanced rearrangements were identified as more complex than previously appreciated [8]. In the same way, extreme balanced germline chromothripsis were identified in patients with autism spectrum disorders and other developmental abnormalities $[9,10]$. Also, chromothripsis was observed in healthy subjects [11, 12] as well as in prenatal diagnosis [13]. Some studies reported the possible reversibility of chromothripsis [14] and its potential curative effect [15]. Accumulating data on familial chromothripsis validated the notion of the heritability of some chromothripsis rearrangements.

Precise analysis of breakpoint junction sequences have indicated that the re-assembly of DNA fragments was driven by recombination-based mechanism such as classical non-homologous end joining (c-NHEJ) or alternative form of end joining (alt-EJ), operating in all phases of the cell cycles and working independently of micro-homologies but potentially error-prone [16-19]. Since the end-joining process mediates the formation of reciprocal translocations and complex three-way translocations, Kloosterman et al. [20] suggested that a similar cascade mechanism could operate in the creation of the derivative complex chromosomes found in constitutional chromothripsis.

Concerning the shattering of chromosome segments, multiple DBSs can arise from various exogenous sources such as ionizing radiation, free radicals, environmental toxins or chemotherapeutic drugs [21]. Even cannabis exposure has been associated with chromothripsis occurrence [22]. Other exogenous causal factors might be certain viral integration such as human papillomavirus (HPV) that can promote genomic instability and multiple DNA breaks [23]. Analysis of the aetiology of chromothripsis has also led to the identification of several cellular mechanisms capable of initiating chromothripsis process. Tubio and Estivill [24] proposed that chromothripsis might be caused by abortive apoptosis. Whereas apoptosis was considered as an irreversible cascade of extensive chromatin fragmentations leading to cell death, a small subset of cells could undergo a restricted form of apoptosis and thus survive. The partial DNA fragmentation could be restricted to regions of high chromatin accessibility. The subsequent DNA repair might be accomplished through a fast and incorrect repair process, promoting the emergence of chaotic chromosomal rearrangement $[16,25]$.

Since many examples of chromothripsis rearrangements affect chromosome ends, it has been proposed that chromothripsis could also arise via telomere attriction $[2,26]$. Indeed, uncapped chromosome-ends are prone to fusion, leading to the formation of dicentric chromosomes [27]. During mitosis, this telomere crisis can yield complex rearrangements through breakage-fusion-bridge (BFB) cycles [28]. Several studies have suggested the association between chromothripsis and the occurrence of BFBs [26, 29]. By examining the fate of dicentric human chromosomes, Maciejowski et al. [30] evidenced the formation of chromatin bridges connecting daughter cells. These bridges can undergo nuclear envelope rupture and nucleolytic attack by cytoplasmic TREX1 exonuclease, causing in the restricted area of the bridge, chromothripsis-like rearrangements frequently associated with local hypermutations known as kataegis [30, 31].

Other proposed models suggest that replication stress and mitotic error could synergize to induce chromosomal instability and chromothripsis occurrence $[16,32,33]$ or that premature chromosome condensation (PCC) induced by the fusion of an interphasic cell with a metaphasic cell could initiate chromothripsis, leading to incomplete replication and subsequent partial pulverization of chromosomes [34].

The emergence of chromothripsis has also been strongly associated with dysregulation or loss of p53 tumour suppressor genes. Known as the guardian of the genome, p53 plays a major role in maintaining genome stability by mediating cell cycle arrest, apoptosis and cell senescence in response to DNA damages [35, 36]. The potential implication of p53 pathways in chromothripsis occurrence was postulated by Rausch et al. [37] after the discovery of a striking correlation between germline p53 mutations (Li-Fraumeni syndrome) and chromothripsis patterns in patients with Sonic-Hedgehog medulloblastoma brain tumors. These findings led the authors to propose that germline p53 mutations could either predispose cell to catastrophic DNA rearrangements or facilitate cell survival after these catastrophic events.

An attractive mechanistic explanation to link all these causal processes with the confined nature of damages created during chromothripsis, is that the implicated chromosome(s) can be incorporated into a micronucleus 


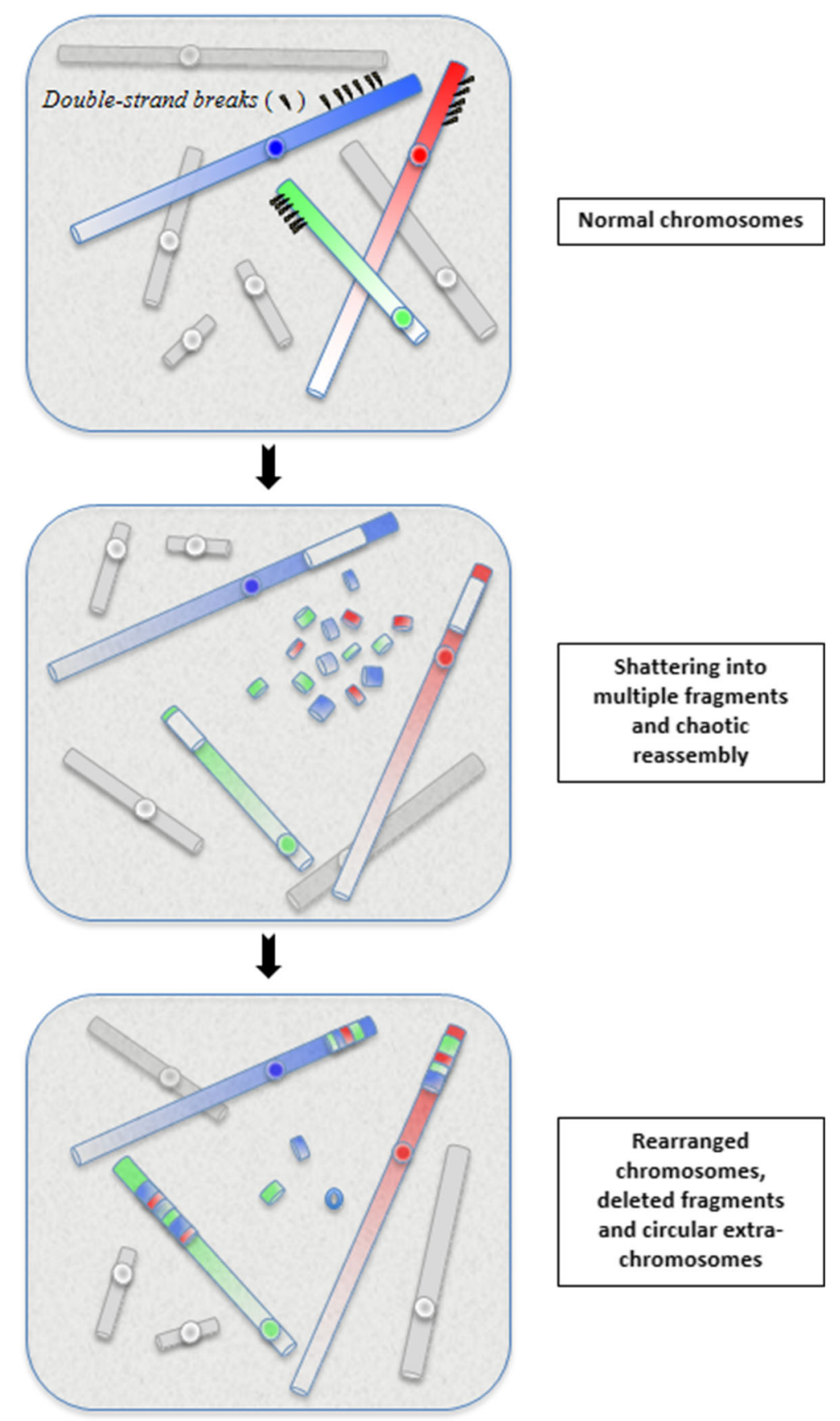

Fig. 1 The concept of chromothripsis: during a one-step catastrophic event, multiple double-strand breaks occurred, restricted to a simple chromosomal segment or to a few closed chromosome domains, leading to the pulverization of chromosomal fragments. This shattering can produce tens to hundreds DNA fragments. Most of them are stitched back together by non-homologous end-joining (NHEJ), resulting in chaotic derivative chromosome(s), whereas some are lost or combined in small circular extra-chromosomes

in which chromothripsis-related damages will occur. Micronuclei are generally considered as passive indicators of chromosomal instability [38]. Crasta et al. [39] provided the first experimental evidence on this mechanism by the generation of micronuclei in several human cell lines and the subsequent observation of extensive genomic rearrangements during the cell cycles following the formation of micronuclei. Micronuclei display a double-membrane similar to regular nuclei, but micronuclei often undergo defective nuclear envelope assembly and the number of nuclear pore complexes (NPCs) is often inadequate. Recently, Liu et al. [40] showed that only "core" nuclear envelope proteins assemble efficiently around lagging chromosomes whereas "non-core" nuclear 
envelope proteins, especially NPCs, do not. This situation leads to a defect in the micronuclear import of essential components for DNA repair and replication, and consequently to reduced functioning in micronuclei. The chromatin sequestrated in micronuclei can undergo defective replication, resulting in the formation of complex rearranged chromosomes [41]. Micronuclei may persist in daughter cells over several cell cycles before being eliminated or reincorporated into the regular nucleus [42]. An additional pathway for the occurrence of DNA damages in micronuclei is the irreversible breakdowns of their membranes during interphase. Zhang et al. [43] proposed that membrane rupture enables enzymes such as endonucleases or topoisomerases to act aberrantly on micronuclear chromosome fragments. The entry of the cell into mitosis while the micronucleus is still undergoing DNA replication will result in micronuclear DNA pulverization due to premature chromosome compaction, and the subsequent chaotic reassembly of chromosome fragments [39, 44].

Using an elegant in vitro model to specifically induce mis-segregation of the $\mathrm{Y}$ chromosome, Ly et al. [45] observed frequent $\mathrm{Y}$ chromosome sequestration into micronuclei, followed by shattering and incorrect reassembly of $\mathrm{Y}$ chromosome fragments through 3 consecutive cell cycles. By using inhibitor of DNA repair, the authors demonstrated that NHEJ mechanism was not efficient in the micronucleus, but operated during the subsequent interphase, after the incorporation of $\mathrm{Y}$ chromosome fragments in a daughter nucleus.

These micronucleus-based models have the potential to explain many features of chromothripsis, especially how such massive damages can be confined to one or just a few chromosomal segments [46].

\section{Chromoanasynthesis}

As investigations on the aetiology of chromothripsis events progressed, it became clear that the chromothripsis mechanism could not account for all the phenomenon of chaotic and rapid genomic rearrangements. Indeed, a number of complex rearrangements with duplication and triplication cannot be explained by NHEJ-mediated repair mechanisms. This led to the proposal that chaotic rearrangements could also result from another one-off cellular event in which there are copy number increases. This distinct process has been identified and named chromoanasynthesis, for chromosome reconstitution [47]. Although its molecular mechanism differs from that of chromothripsis, its biological consequences are similar, with the rapid formation of highly remodelled chromosomes. To date, most of patients with chromoanasynthesis-mediated rearrangements display developmental delay, intellectual disability and dysmorphic features, but individuals with relatively mild phenotypic effects have also been described [48, 49].
Chromoanasynthesis is considered to be a replicationbased complex rearrangement process that involves serial fork stalling and template switching (FoSTeS) or microhomology-mediated break-induced replication (MMBIR) mechanisms [50, 51].

Numerous exogenous or endogenous agents can create conditions of replication stress by interfering with the progression and the stability of the replication fork [52, 53]. In a stressing situation, when the replication forks stall or pause in the vicinity of DNA lesions, fragile sites, cluster of tightly bound proteins or structural domains that are difficult to replicate, such replication stress may lead to aberrant replication with the use of alternate error-prone DNA repair mechanisms such as FoSTeS and MMBIR that lead to the formation of complex structural changes and copy number variations [54].

In the models of FoSTeS and MMBIR, the lagging DNA-strand end can serially disengage and switch to another nearby template. DNA would then be copied by another active replication fork. The new template strand is not necessarily adjacent to the initial replication fork, but in 3D physical proximity. Multiple fork disengaging, and strand invasions can occur before the resumption of replication on the original template (Fig. 2).

Like chromothripsis, chromoanasynthesis events involve a combination of structural rearrangements. However, the occurrence of localized multiple copy-number changes, particularly region-focused duplication and triplication and short stretches of micro-homologies at the breakpoint junctions, are both the hallmarks of replication-based mechanism with iterative template switches and define the chromoanasynthesis phenomenon. In addition, a high incidence of marker chromosomes has been reported in patients with chromanasynthesis disorders $[55,56]$.

Molecular situations responsible of replication fork stalling are numerous and a variety of cellular events may trigger the genome instability underlying chromanasynthesis events. All environmental insults and physiological pathway alterations that compromise genome stability, may potentially give rise to replication stress and subsequent chromoanasynthesis occurrence [57].

Such replication-based mechanisms do not necessarily require micronucleus formation to explain the occurrence of massive chromosomal rearrangements. However, the micronucleus-mediated models proposed for chromothripsis provide attractive cellular explanation also for chromoanasynthesis phenomenon.

\section{Chromoplexy}

A third type of massive rearrangement has been evidenced in prostate cancer. Termed chromoplexy [58], for chromosome restructuring, this phenomenon is characterized by the interdependent occurrence of multiple inter-and intra-chromosomal translocations and deletions (Fig.3). 


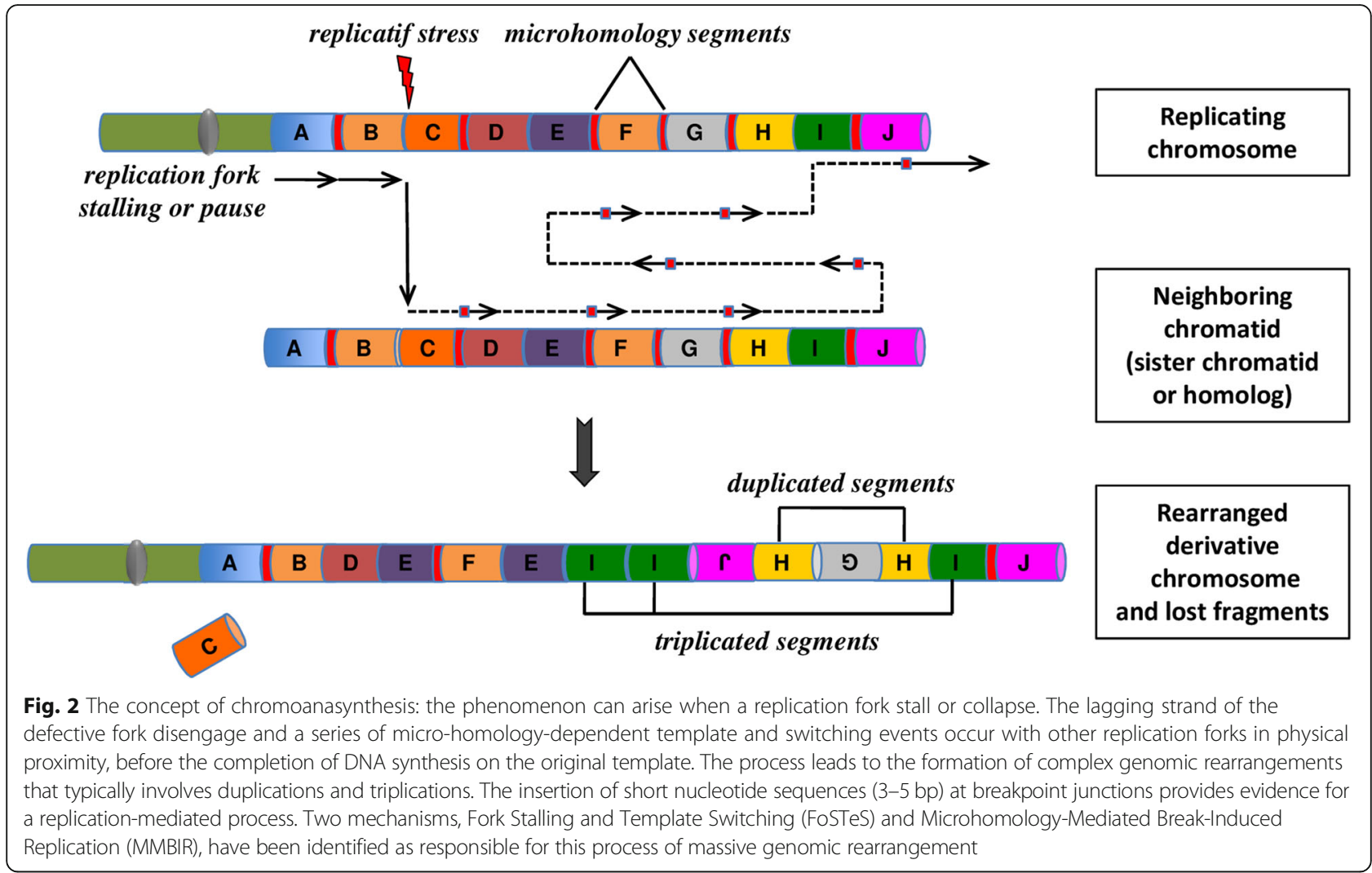

The analysis of breakpoint sequences indicated that all the partner fragments involved in these serial rearrangements originate from DSB and exhibit deletion at the fusion junctions of chained rearrangements. Computational simulations revealed that chromoplexy events arise through a single catastrophic event.

These chains of rearrangements, numbering from 3 to over 40 and involving up to 7 chromosomes in a single chain, occur in a large majority of the prostate cancer studied. In contrast to chromothripsis, chromosomal rearrangements present little or no copy number alterations.

The involvement of the TMPRSS2-ERG gene fusion $(\mathrm{EST}+)$ in the complex event, suggest chromoplexy may arise from the same transcriptional-mechanism driven by the androgen receptor (AR) that induce TMPRSS2-ERG fusion. Thus, the nuclear co-localization of transcriptional sites could be mediated by the co-localization of androgen receptor [59]. AR-mediated transcription has been implicated in the occurrence of DSBs. In the context of chromoplexy-related process, the AR, by coordinating the induction of clustered DSBs, might effectively promote the formation of chained rearrangements within a restricted nuclear domain.

Sequence analyses of prostate tumour specimens revealed distinctive patterns of chromoplexy. Tumour harbouring oncogenic ETS fusion (ETS+) produced more inter-chromosomal rearrangements whereas tumour with a deletion of CHD1, a putative tumour suppressor gene, showed an excess of intra-chromosomal chained rearrangements. Chromoplexy could simultaneously dysregulate multiple cancer genes across the genome and contribute to the aggressive evolution of high-grade prostate cancers. The detection of similar chained rearrangements in cases of lung cancers, melanomas or neck cancers suggests that chromoplexy could occur in a larger spectrum of cancers [60].

Interestingly, these data concerning the chromoplexy process also supports the punctuated equilibrium model of cancer evolution [61].

\section{Factors operating in chromoanagenesis}

What drives the cell in the choice of a DNA repair process? The question is particularly relevant since repair outcomes don't always seem to be in line with the ultimate goal of preserving genome integrity. Firstly, this choice must be dictated by the cell-cycle position. NHEJ can occur at any stage of the cell cycle. In contrast, replicative repair pathways operate only during $S$-phase and eventually in G2. Of particular importance are the number of DSBs and the energy required by the cell to manage repairs on a short timescale. High loads of DSBs can rapidly saturate the repair machinery. Gudjonsson et al. [62] demonstrated that more than 20 DSBs can alter classical error-free repair pathways such as homologous 


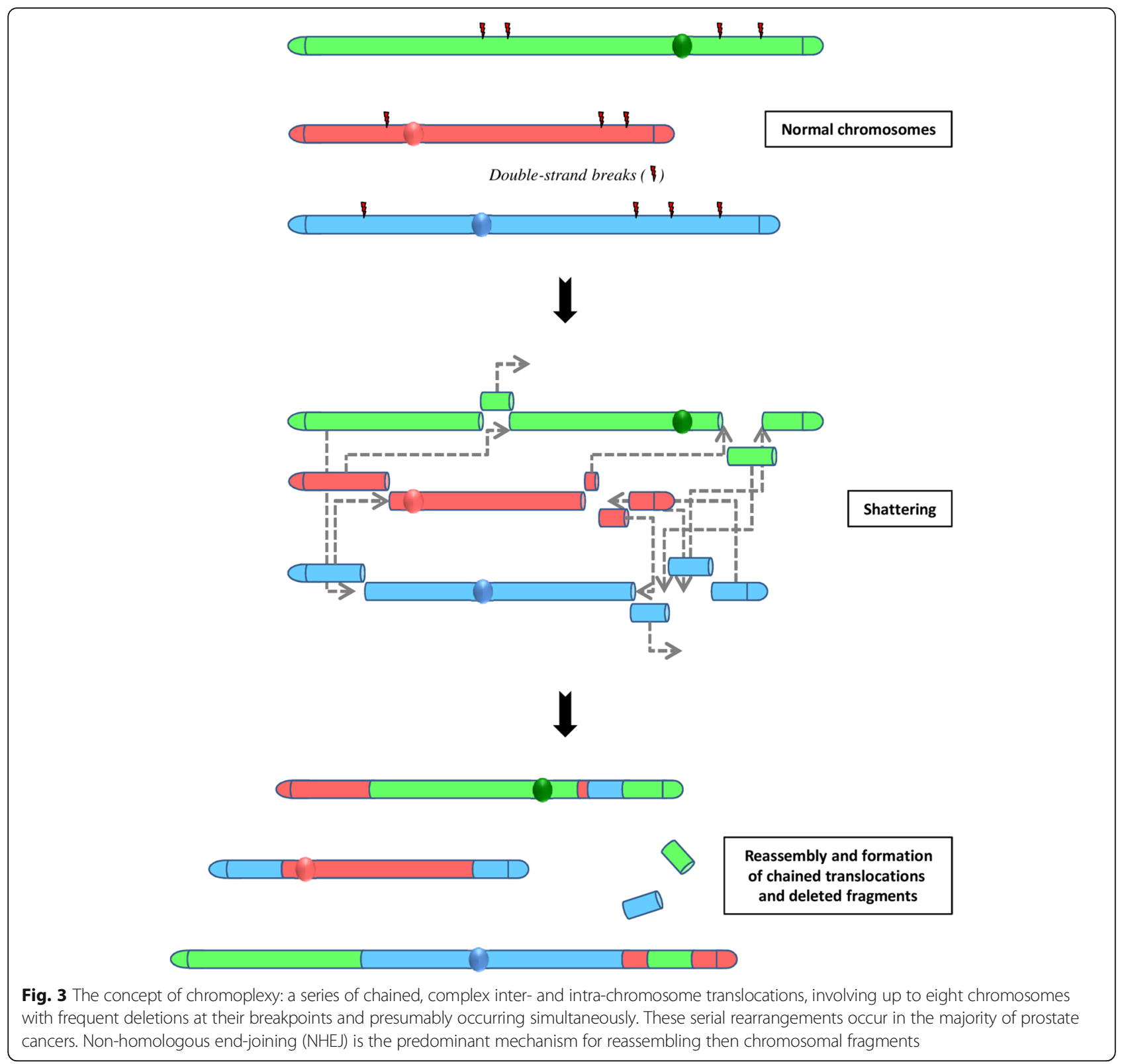

recombination (HR), thus leading the cell to engage in faster but error-prone repair processes such as c-NHEJ or alt-EJ. It is also considered that the complexity of DSBs and the accompanying chemical alterations are determinants for the cellular choice of DSB repair pathway [63]. Specific sequence features may stimulate or simply predispose to the occurrence of complex genomic reassociations. Analyses at the junction points have evidenced the recurrent presence of unusual DNA sequences such as low-copy repeats (LCRs) or tandem repeats such as Alu or LINE sequences $[5,64]$. In some chromothripsis and chromoanasynthesis breakpoints, the frequency of LCRs or LINEs is significantly higher than the genome-wide average [65]. These repetitive sequences constitute points of genomic instability and may serve as substrates for chromosomal rearrangements [66, 67]. For instance, the relatively long micro-homology (20-40 bp) shared by Alu sequences may facilitate template switching in replicative repair processes [68]. Other particular elements, such as palindromic sequences, have the potential to form distinct secondary structures, which are able to impede replication and stimulate DSBs [69]. In chromoanasynthesis investigation, Liu et al. [47] demonstrated that complex genome architecture such as hairpin structure, palindromic sequences or low-copy repeat clusters may confuse the DNA replication machinery and exacerbate serial template switching events driven by FoSTeS and MMBIR. 
The chromatin structure also regulates DSB repair through histone modifications and nucleosome remodeling within approximately $50 \mathrm{~kb}$ on each side of DSBs, in order to promote the formation of an open, relaxed chromatin structure at the DSBs and to facilitate loading of DNA repair proteins [70,71]. With regards to this process, the compact heterochromatin is less accessible and potentially less sensitive than euchromatin to DNA damaging agents that initiate DSBs. A plausible connection between chromothripsis and fragile sites has also been postulated [72]. Fragile sites contribute to genomic instability [73] and thus they can be preferential sites for the multiple breakage events observed in chromothripsis and chromoanasynthesis events.

Recent reports provided evidence that transposable elements can serve as drivers in the formation of chromoanagenesis by compromising the genomic stability and facilitating chromatin conformation changes and DNA breaks [74, 75]. The presence of transposable elements within the genome is currently associated with chromosome restructuring [76], genetic disorders [77] and chromosome evolution [78]. The abundance of these elements in the human genome provides numerous potential substrates for microhomology-mediated template switching and chromoanasynthesis occurrence.

Lastly, one could speculate than the genomic architectural feature is a determining factor in chromoanagenesis emergence. The detection of chromothripsis on unique chromosomal regions has suggested that shattering and reassociation might occur when chromosomes are highly condensed, i.e. during mitosis. However, examples of constitutional chromoanagenesis-compatible events implicating several chromosomes indicate that the phenomenon may preferentially arise during interphase when chromosomes are relaxed throughout the nucleus.

The general consensus is that interphase chromosomes are compartmentalized in discrete but dynamic nuclear territories that could confine intranuclear processes to a limited space $[79,80]$. This view could imply the spatial proximity of chromosomes involved in chromothripsis and their proximity with potential discrete domains that cluster DNA repair factors [81, 82].

Accumulating date from chromosome configuration capture (3C)-based approaches and the analysis of topologically associated domains (TADs) provide information on cell-to-cell architecture variability and indicate how structural rearrangements in the layout of genes and their regulatory sequences can lead to ectopic gene interactions and altered gene expression [83-85]. HI-C experiments and TADs analysis performed on induced pluripotent stem cells (iPSCs) derived from a patient with a de novo germline chromothripsis have allowed to evidence how the chromothripsis rearrangements led to gene dysregulation and contribute to the patient's complex congenital phenotype [86]. The occurrence of massive chromosome rearrangements can lead to deleterious genomic configurations but also to genetic novelty, with the formation of stable and inheritable rearranged genomic constitution. In addition to 3D genome organization, another important factor could be the movement of chromatin within the nucleus. Certain genes seem to have preferential contacts with their neighbours in a phenomenon termed "chromosome kissing" [87]. Local movements of DSBs have been evidenced in yeast [88] and Drosophilia [89] where DSBs within heterochromatic domains have to move to a more euchromatic environment to be repaired. Certain experiments in mammalian cells have suggested limited mobility for DSBs [90], whereas others have shown the intranuclear repositioning of derivative chromosomes and their normal counterparts in translocation cell lines [91]. To reconcile this large range of data, Dion and Gasser [92] proposed that different types of DNA damages lead to different modes of nuclear movements, depending on how the lesion is repaired. The localization of chromothripsis restricted to a single chromosome segment or to a small nuclear territory could establish the upper limit of tolerance of the phenomenon by the cell. Further works are needed to discover if chromatin mobility and its proximity with some elements of nuclear architecture (such as nucleolus, nuclear matrix, lamina) could be a limitative factor in case of accumulation of DSBs.

Whatever parameters are implicated in the emergence of chromoanagenesis events, the high probability of error in repair processing as well as in replication mechanisms suggests that cells have developed tolerance for sequence modifications at the breakpoint junctions. The logic underlying the emergency of chromoanagenesis events could be more the preservation of genomic stability than genomic integrity.

\section{Chromoanagenesis in cancer}

Chromothripsis was originally described in a case of chronic lymphocytic leukemia in a female patient displaying 42 somatic acquired structural rearrangements on the long arm of chromosome 4 [2] The discovery of such a pattern of massive interchromosomal rearrangements was made by combining next-generation paired-end sequencing and single nucleotide polymorphism (SNP) assays. To date, chromothripsis has now been described in a broad spectrum of human cancers including neuroblastoma, medulloblastoma, myeloma, retinoblastoma, colorectal cancers, or hematologic malignancies [93-99]. A survey of 4934 cancers indicated that chromothripsis was found in $5 \%$ of all samples, with incidences ranging from $0 \%$ in head carcinoma to $16 \%$ in glioblastoma [100]. A large analysis of 8227 cancers revealed the occurrence of chromothripsis-like massive rearrangements in 1 
to $2 \%$ of the sample [101]. Through a large-scale analysis of more 22.000 tumoral array data sets covering 132 cancer types, Cai et al. [102] evidenced the heterogeneity of the genome aberrations patterns associated with chromothripsis-like events. Altogether, these data provide evidence that at least 2 to $4 \%$ of all human cancers involve chromothripsis events, affecting one or several chromosomes. Glioblastoma and bone tumours appear to be the most affected types of cancer with up to 39 and $25 \%$ of chromothripsis [103].

In all cases, chromothripsis is associated with aggressive forms of cancer and poor patient survival [104, 105]. According to the type of cancer, specific chromosomes have been identified to be more sensitive to chromoanagenesis events $[98,102]$. Thus, chromothripsis was more frequently detected in genomic regions containing critical gene for the DNA repair, the cell cycle regulation or the proliferation [106]. In some tumoral chromothripsis, the chaotic reorganization may lead to the generation of circular, extra double-minute chromosome markers that often include oncogenes and are frequently amplified [37, 107]. This may contribute to substantial changes in copy number state as well as the chromosomal instability in tumoral cells [1]. Another example of the contribution of chromanagenesis to tumoral evolution is the formation of neochromosomes, giant extra-chromosomes found in 3\% of cancers, which associates chromoanagenesis events and BFB cycles [108, 109]. However, chromothripsis was also observed in uterine leiomyomas, a common and low-malignant smooth-muscle tumour, indicating that chromothripsis does not systematically have a dramatic oncogenic effect [110].

TP 53 mutations have been associated with chromothripsis in medulloblastoma and leukemia [37, 111]. High prevalence of chromothripsis events was also reported in patients with Ataxia Talangiectasia [99], indicating that alteration affecting other essential pathway for the maintenance of genome stability and cell cycle progression, such as ATM function, can also trigger chromothripsis occurrence. Also, studies of retinoblastoma progression indicated that chromothripsis can initiate tumorogenesis by inactivating a tumour suppressor gene [97]. Complex breakpoints in cancers may also exhibit significant array of short sequences derived from distinct loci, suggesting replication-based mechanisms consistent with chromanasynthesis events [112]. Collectively, these data suggest that cancer-associated chromoanagenesis rearrangements are more complex and subtle than previously envisaged, with the creation of various oncogene lesions, loss or disruption of tumour suppression genes and the construction of oncogenic fusions. Alterations in oncogenes or tumour suppressors that destabilize the genome can induce chromosome lagging and micronuclei formation. The formation of micronuclei containing whole chromosome(s) or chromosome fragments has been documented for many years as a frequent hallmark of genome instability in cultured tumoral cells [113], but we do have precise information on their real in vivo frequency.

The long-standing paradigm that genome alterations in cancer arise through the progressive accumulation of mutation has been deeply challenged by the discovery of chromoanagenesis events that might constitute major mutational gamers. Thus, in pancreatic cancer, the observation that two-thirds of tumours harboured complex chromothripsis-like patterns has contributed to the notion that pancreatic cancer progression was not gradual [98]. The concept that cancer genome can evolve in rapid bursts is consistent with the evolutionary model of punctuated equilibrium (see chapter below).

\section{Chromanagenesis and evolution}

Beyond the impact of chromoanagenesis events as pathogenic mechanisms, an interesting question is the potential driving role of these phenomenon in species evolution. The occurrence of chromoanagenesis event appears to be in good agreement with macroevolution models such as the "hopeful monster" model [114] or the "punctuated equilibrium" theory [115] proposed as a complement to phyletic gradualism. These models postulated that species undergo little variations during most of their evolutionary history, interrupted by rare but abrupt bursts of change leading to the subsequent emergence of new species. During the last decade, accumulated data have demonstrated how genetic and chromosomal alterations can have huge impacts in developmental evolution. Numbers of studies have documented punctuated equilibrium and hopeful monsters in various species, introducing the notion of "transgressive segregations" for the generation and the rapid fixation of new genotypes in population. Prominent models argue that chromosomal rearrangements reduce gene flow through their suppressive effect on recombination [116]. Complex rearrangements such as chromoanagenesis events may modify gene position relative to replication origins or lead to the generation of new linkage gene block or new chimeric genes. Several models of chromosomal speciation are thus based on the existence of gametic barriers resulting from the fixation of various genomic rearrangements in a population [117]. Thus, in the gibbon genome, the insertion of a retro-transposon named LAVA in genes implicated in cell cycle progression and chromosome segregation appears to be at the origin of a high rate of chromothripsis-like rearrangements leading to the accelerated evolution of the gibbon karyotype and the emergence of different gibbon lineages $[118,119]$. Another example of genome reshuffling and speciation driven by massive chromosome rearrangements 
is the extensive chromosome reshuffling observed in the marsupial family Macropodidae, with numerous interchromosomal rearrangements [120]. In 2007, Crombac and Hogeweg [121] demonstrated that genome restructuring mediated by massive chromosomal rearrangements was a beneficial operator for shorty-term adaptations to a new environment. Chromoanagenesis events as processes for rapid and profound genomic modification could be regarded as credible molecular mechanisms for evolutionary changes.

\section{Conclusion}

Undoubtedly, chromothripsis, chromanasynthesis and chromoplexy are among the most unexpected biological discoveries made from the high-resolution genome analysis. The identification of these 3 unanticipated catastrophic phenomenon has deeply modified our perception of the genesis and the aetiology of complex genomic rearrangements. The investigation of this new class of genomic alterations has also provided new and important insights on the mechanisms connecting defective cell cycle progression with cellular stress and genomic instability, the role of genome maintenance pathways and the capacity of cells to manage such crisis phenomenon [122, 123]. This found expression in the causal link between disordered mitotic progression and the occurrence of complex structural rearrangements via the micronuclei-based process.

All these data support the idea that the occurrence of chromoanagenesis events in the genome is grossly underestimated and that extremely complex but balanced structural rearrangements can be tolerated in human germline and compatible with viability [124]. To date, the existence of chaotic genomic alterations is not restricted to human but there are also documented in other mammalians [118, 120] in plants [125], in nematode Caenorhabditis elegans [126], and Saccharomyces cerevisiae [127], emphasizing the notion that the cellular pathways responsible for generating such highly complex patterns of chromosomal rearrangements are highly conserved.

The identification of chromoanagenesis phenomenon in both cancers and congenital disorders provides a new perception of how genomes can be rapidly altered. Despite a high incidence of cell death during the process, the formation of chaotic genomes could represent a powerful survival strategy for the genome when under crisis, and chromoanagenesis-mediated events could constitute inherent mechanisms for maintaining genome stability and integrity $[128,129]$.

\footnotetext{
Abbreviations

BFB: Breakage-fusion-bridge; DSB: Double-strand break; FoSTeS: Fork stalling and template switching; HPV: Human papillomavirus; HR: Homologous recombination; iPSC: Induced pluripotent stem cell; LCR: Low-copy repeat; MMBIR: Microhomology-mediated break-induced replication; NHEJ: Nonhomologous end joining; NPC: Nuclear pore complex; PCC: Premature chromosome condensation; TAD: Topologically associated domain
}

Acknowledgements

The author thanks Little Anne for her support and attention.

Funding

Work in the unit of Chromosomal Genetics is supported by the $\mathrm{CHU}$ research platform CHROMOSTEM (http://www.chu-montpellier.fr/fr/chercheurs/ plateformes/les-plateformes-recherche/chromostem/).

\section{Availability of data and materials}

The datasets used and analysed in this review are available from the corresponding author on reasonable request.

\section{Authors' contributions}

FP: review design, data analysis, manuscript preparation and writing. The author read and approved the final manuscript.

\section{Ethics approval and consent to participate}

Not applicable.

\section{Consent for publication}

Not applicable.

\section{Competing interests}

The author declares that he/she has no competing interests.

\section{Publisher's Note}

Springer Nature remains neutral with regard to jurisdictional claims in published maps and institutional affiliations.

Received: 18 December 2018 Accepted: 17 January 2019

Published online: 11 February 2019

\section{References}

1. Holland AJ, Cleveland DW. Chromoanagenesis and cancer: mechanisms and consequences of localized, complex chromosomal rearrangements. Nat Med. 2012;18:1630-8.

2. Stephens PJ, Greenman CD, Fu B, Yang F, Bignell GR, Mudie LJ, et al. Massive genomic rearrangement acquired in a single catastrophic event during cancer development. Cell. 2011;144:27-40.

3. Maher CA, Wilson RK. Chromothripsis and human disease: piecing together the shattering process. Cell. 2012;148:29-32.

4. Korbel JO, Campbell PJ. Criteria for inference of chromothripsis in Cancer genomes. Cell. 2013;152:1226-36.

5. Kloosterman WP, Gurvey V, van Roosmalen M, Duran KJ, de Bruijn E, Bakker SCM, et al. Chromothripsis as a mechanism driving complex de novo structural rearrangements in the germline. Hum Molec Genet. 2011;20:1916-24.

6. Chiang C, Jacobsen JC, Ernst C, Hanscom C, Heilbut A, Blumenthal I, et al. Complex reorganization and predominant non-homologous repair following chromosomal breakage in karyotypically balanced germline rearrangements and transgenic integration. Nat Genet. 2012;44:390-998.

7. Faulin Gamba B, Richieri-Costa A, Costa S, Rosenberg C, Ribeiro-Bicudo LA Chromothripsis with at least 12 breaks at 1 p36.33-p35.3 in a boy with multiple congenital anomalies. Mol Gen Genomics. 2015;290(6):2213-6.

8. Weckselblatt B, Hermetz KE, Rudd MK. Unbalanced translocations arise from diverse mutational mechanisms including chromothripsis. Genome Res. 2015;2(7):937-47.

9. Anderson SE, Kamath A, Pilz DT, Morgan SM. A rare example of germ-line chromothripsis resulting in large genomic imbalance. Clin Dysmorphol. 2016;25(2):58-62.

10. Collins RL, Brand H, Redin CE, Hanscom C, Antolik C, Stone MR, et al. Defining the diverse spectrum of inversions, complex structural variation, and chromothripsis in the morbid human genome. Genome Biol. 2017. https://doi.org/10.1186/s13059-017-1158-6.

11. de Pagter MS, van Roosmalen MJ, Baas AF, Renkens I, Duran KJ, et al. Chromothripsis in healthy individuals affects multiple protein-coding genes and can result in severe congenital abnormalities in offspring. Am J Hum Genet. 2015;96(4):651-6.

12. Bertelsen B, Nazaryan-Petersen L, Sun W, Mehrjouy MM, Xie G, Chen W, et al. A germline chromothripsis event stably segregating in 11 individuals through three generations. Genet Med. 2015;18:494-500. 
13. Macera MJ, Sobrino A, Levy B, Jobanputra V, Aggarwal V, et al. Prenatal diagnosis of chromothripsis, with nine break characterized by karyotyping, FISH, microarray and whole-genome sequencing. Prenatal Diagn. 2015;35(3):299-301

14. Bassaganyas L, Bea S, Escaramis G, Tornador C, Salaverria I, et al. Sporadic and reversible chromothripsis in chronic lymphocytic leukemia revealed by longitudinal genomic analysis. Leukemia. 2013;27:2376-424

15. McDermott DH, Gao JL, Liu Q, Siwicki M, Martens C, Jacobs $P$, et al. Chromothripsis cure of WHIM syndrome. Cell 015. 160(4):686-99.

16. Jones MJK, Jallepalli PV. Chromothripsis: chromosomes in crisis. Dev Cell. 2012:23:908-17

17. Iliakis G, Murmann T, Soni A. Alternative end-joining repair pathways are the ultimate backup for abrogated classical non-homologous end-joining and homologous recombinatioin repair: implications for the formations of chromosome translocations. Mutat Res Genet Toxicol Environ Mutagen. 2015;793:166-75.

18. Masset $H$, Hestand MS, Van Esch $H$, Kleinfinger $P$, Plaisancié J, Afenjar A, et al. A distinct class of chromoanagenesis events characterized by focal copy number gains. Hum Mutat. 2016;37(7):661-8.

19. So A, Le Guen T, Lopez BS, Guirouilh-Barbat J. Genomic rearrangements induced by unscheduled DNA double strand breaks in somatic mammalian cells. FEBS J. 2017;284:2324-44

20. Kloosterman WP, Cuppen E. Chromothripsis in congenital disorders and cancer: similarities and differences. Curr Opin Cell Biol. 2013;25:341-8.

21. Morishita M, Muramatsu T, Suto Y, Hirai M, Konishi T, Hayashi S, et al. Chromothripsis-like chromosomal rearrangements induced by ionizing radiation using proton microbeam irradiation system. Oncotarget. 2016;7(9):10182-92.

22. Reece AS, Hulse GK. Chromothripsis and epigenomics complete causality criteria for cannabis-and addiction-connected carcinogenicity, congenital toxicity and heritable genotoxicity. Mutat Res. 2016;789:15-25.

23. Akagi K, Li J, Broutian TR, Padilla-Nash H, Xiao W, Jiang B, et al. Genomewide analysis of HPV integration in human cancers reveals recurrent, focal genomic instability. Genome Res. 2016;24:185-99.

24. Tubio JMC, Estivill X. When catastrophe strikes a cell. Nature. 2011;470:476-7.

25. Ciavarra G, Zacksenhaus E. Multiple pathways counteract cell death induced by RB1 loss: implications for cancer. Cell Cycle. 2011;15:1533-9.

26. Ernst A, Jones DTW, Maass KK, Rode A, Deeg Kl, Jebaraj BMC, et al. Telomere dysfunction and chromothripsis. Int J Cancer. 2016;138(12):2905-14.

27. Hackett JA, Feldser DM, Greider CW. Telomere dysfunction increases mutation rate and genomic instability. Cell. 2001;106(3):275-86.

28. Gisselsson D, Jonson T, Petersen A, Strombeck B, Dal Cin P, Hoglund M, et al. Telomere dysfunction triggers extensive DNA fragmentation and evolution of complex chromosome abnormalities in human malignant tumors. Proc Natl Acad Sci U S A. 2001;98:12683-8.

29. Sorzano CO, Pascual-Montano A, Sanchez de Diego A, Martinez AC, van Wely KH. Chromothripsis: breakage-fusion-bridge over and over again. Cel Cycle. 2013;12(13):2016-23.

30. Maciejowski J, Li Y, Bosco N, Campbell PJ, de Lange T. Chromothripsis and Kataegis induced by telomere crisis. Cell. 2015;163:1641-54.

31. Maciejowski J, de Lange T. Telomeres in cancer: tumour suppression and genome instability. Nat Rev Mol Cell Biol. 2017;18(3):175-86

32. Mardin BR, Drainas AP, Waszak SM. A cell-based model system links chromothripsis with hyperploidy. Mol Syst Biol. 2015. https://doi.org/10. 15252/msb.20156505.

33. Passerini V, Ozeri-Galai E, de Pagter M, Donnelly N, Schmalbrock S, Kloosterman WP, et al. The presence of extra chromosomes leads to genomic instability. Nat Commun. 2016. https://doi.org/10.1038/ ncomms 10754 .

34. Terzoudi Gl, Karakosta M, Pantelias A, Hatzi VI, Karachristou I, Pantelias G. Stress induced by premature chromatin condensation triggers chromosome shattering and chromothripsis at DNA sites still replicating in micronuclei or multinucleate cells when primary nuclei enter mitosis. Mutat Res Genet Toxicol Environ Mutagen. 2015;793:185-98.

35. Vogelstein B, Lane D, Levine AJ. Surfing the p53 network. Nature. 2000; 408(6810):307-10

36. Fridman JS, Lowe SW. Control of apoptosis by p53. Oncogene. 2003:22:9030-40.

37. Rausch T, Jones DTW, Zapatka M, Stütz AM, Zichner T, Weischenfeldt J, et al. Genome sequencing of pediatric medulloblastoma links catastrophic DNA rearrangements with TP53 mutations. Cell. 2012;148:59-71.

38. Terradas M, Martin M, Genesca A. Impaired nuclear functions in micronucle results in genome instability and chromothripsis. Arch Toxicol. 2016;90:2657-67.
39. Crasta K, Ganem NJ, Dagher R, Lanterman AB, Ivanova EV, Pan Y, et al. DNA breaks and chromosome pulverization from errors in mitosis. Nature. 2012;482:53-8.

40. Liu S, Kwon M, Mannino M, Yang N, Renda F, Khodjakov A, et al. Nuclear envelope assembly defects link mitotic error to chromothripsis. Nature. 2018:561(7724):551-5

41. Terradas M, Martin M, Tusell L, Genesca A. Genetic activities in micronuclei: is the DNA entrapped in micronuclei lost for the cell ? Mutat Res. 2010;705:60-7.

42. Huang $Y$, Jiang $L$, Yi Q, Lv L, Wang Z, Zhao $X$, et al. Lagging chromosomes entrapped in micronuclei are not "lost" by cell. Cell Res. 2012;22:932-5.

43. Zhang CZ, Spektor A, Cornils H, Francis JM, Jackson EK, Liu S, et al. Chromothripsis from DNA damage in micronuclei. Nature. 2015;522:179-84.

44. Luijten MNH, Lee JXT, Chen S, Crasta K. Generation of micronuclei and detection of chromosome pulverization. Methods Mol Biol. 2018;1769:183-95.

45. Ly P, Teitz LS, Kim DH, Shoshani O, Skaletsky H, Fachinetti D, et al. Selective $Y$ centromere inactivation triggers chromosome shattering in micronuclei and repair by non-homologous end joining. Nature Cell Biol. 2017:19:68-77.

46. Russo A, Degrassi F. Molecular cytogenetics of the micronucleus: still surprising. Mutat Res. 2018;836:36-40.

47. Liu P, Erez A, Sreenath Nagamani SC, Dhar SU, Kolodziejska KE, Dharmadhikari $A V$, et al. Chromosome catastrophes involve replication mechanisms generating complex genomic rearrangements. Cell. 2011;146:889-903.

48. Suzuki E, Shima H, Toki M, Hanew K, Matsubara K, Kurahashi H, et al. Complex X-chromosomal rearrangements in two women with ovarian dysfunction: implications of chromothripsis/chromoanasynthesis-dependant and-independent origins of complex genomic alterations. Cytogenet Genom Res. 2016;150:86-92.

49. Fukami M, Kurahashi $\mathrm{H}$. Clinical consequences of chromothipsis and other catastrophic cellular events. Methods Mol Biol. 2018;1769:21-33.

50. Lee JA, Carvalho CM, Lupski JR. A DNA replication mechanism for generating nonrecurrent rearrangements associated with genomic disorders. Cell. 2007:131:1235-47.

51. Hastings PJ, Ira G, Lupski JR. A microhomology-mediated break-induced replication model for the origin of human copy number variation. PLoS Genet. 2009;5:e1000327.

52. Branzei D, Foiani $M$. The DNA damage response during DNA replication. Curr Opin Cell Biol. 2005;17(6):568-75.

53. Venkatesan S, Natarajan AT, Hande MP. Chromosomal instability mechanisms and consequences. Mutat Res. 2015;793:176-84.

54. Arlt MF, Wilson TE, Glover TW. Replication stress and mechanisms of CNV formation. Curr Opin Genet Dev. 2012;22:204-10.

55. Grochowski CM, Gu S, Yuan B, Tcw J, Brennand KJ, Sebat J, et al. Marker chromosome genomic structure and temporal origin implicate a chromoanasynthesis event in a family with pleiotropic psychiatric phenotypes. Hum Mutat. 2018;39(7):939-46.

56. Al-Rikabi ABH, Sona Pekova XF, Jancuskova T, Liehr T. Small supernumerary marker chromosome may provide information on dosage-insensitive pericentric regions in human. Curr Genomics. 2018;19(3):192-9.

57. Aguilera A, Gomez-Gonzalez B. Genome instability: a mechanistic view of its causes and consequences. Nat Rev Genet. 2008:9:204-17.

58. Baca SC, Prandi D, Lawrence MS, Mosquera JM, Romanel A, Drier Y, et al. Punctuated evolution of prostate caner genomes. Cell. 2013;153:666-77.

59. Liu W, Lindberg J, Sui G, Luo J, Egevad L, Li T, et al. Identification of novel CHD1-associated collaborative alterations of genomic structure and functional assessment of CHD1 in prostate cancer. Oncogene. 2012;31:3939-48.

60. Shen MM. Chromoplexy: a new category of complex rearrangements in the cancer genome. Cancer Cell. 2013;23(5):657-569.

61. Wang K, Wang Y, Collins CC. Chromoplexy; a new paradigm in genome remodelling and evolution. Asian J Androl. 2013;15:711-2.

62. Gudjonsson T, Altmeyer M, Savic V, Toledo L, Dinant C, Grofte M, et al. TRIP12 and UBR5 suppress spreading of chromatin ubiquitylation at damaged chomosomes. Cell. 2012;150:697-709.

63. Georgakilas AG, O'Neill P, Stewart RD. Induction and repair of clustered DNA lesions: what do we know so far? Radiat Res. 2012;180:100-9.

64. Liu P, Carvalho CMB, Hastings PJ, Lupski JR. Mechanisms for recurrent and complex human genomic rearrangements. Curr Opin Genet Dev. 2012;22:211-20.

65. Poot M. Genes, proteins, and biological pathways preventing chromothripsis. Methods Mol Biol. 2018;1769:231-52.

66. George CM, Alani E. Multiple cellular mechanisms prevent chromosomal rearrangements involving repetitive DNA. Critic Rev Bioch Mol Biol. 2012;47: 297-313. 
67. Weckselblatt B, Rudd MK. Human structural variation: mechanisms of chromosome rearrangements. Trends Genet. 2015;31(10):587-99.

68. Zhang F, Carvalho CM, Lupski JR. Complex human chromosomal and genomic rearrangements. Trends Genet. 2009;25(7):298-307.

69. Kurahashi $H$, Bolor $H$, Kato $T$, Kogo H, Tsutsumi M, Inagaki $H$, et al. Recent advance in our understanding of the molecular nature of chromosomal abnormalities. J Hum Genet. 2009;54:253-60.

70. Peng JC, Karpen GH. Epigenetic regulation of heterochromatic DNA stability. Curr Opin Genet Dev. 2008;18:204-11.

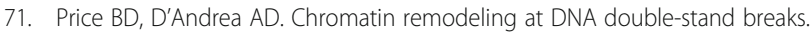
Cell. 2013;152:1344-54

72. Mackinnon RN, Campbell LJ. Chromothripsis under the microscope: a cytogenetic perspective of two cases of AML with catastrophic chromosome rearrangement. Cancer Genet. 2013;206:238-51.

73. Barlow JH, Faryabi RB, Callén E, Wong N, Malhowski A, Chen HT, et al. Identification of early replicating fragile sites that contribute to genome instability. Cell. 2013:152:620-32.

74. Nazaryan-Petersen L, Bertelsen B, Bak M, Jonson L, Tommerup N, Hancks DC, et al. Germline chromothripsis driven by L1-mediated retrotransposition and Alu/Alu homologous recombination. Hum Mutat. 2016:37:385-95.

75. Hancks DC. A role for retrotransposons in chromothripsis. Methods Mol Biol. 2018;1769:169-82

76. Wang L, Rishishwar L, Marino-Ramirez L, Jordan IK. Human population-specific gene expression and transcriptional network modification with polymorphic transposable elements. Nucleic Acids Res. 2017;45:2318-28.

77. Burns KH. Transposable elements in cancer. Nat Rev Cancer. 2017;17:415-24.

78. Klein SJ, O'Neill RJ. Transposable elements: genome innovation, chromosome diversity, and centromere conflict. Chromosom Res. 2018;26:5-23.

79. Cremer T, Cremer C. Chromosome territories, nuclear architecture and gene regulation in mammalian cells. Nat Rev Genet. 2001;2:292-301.

80. Bickmore WA, van Steensel B. Genome architecture: domain organization of interphase chromosomes. Cell. 2013;152:1270-84.

81. Lisby M, Mortensen UH, Rothstein R. Colocalization of multiple DNA doublestrand breaks at a single Rad52 repair Centre. Nat Cell Biol. 2003;5:572-7.

82. Brown JM, Leach J, Reittie JE, Atzberger A, Lee-Prudhoe J, Wood WG, et al. Coregulated human globin genes are frequently in spatial proximity when active. J Cell Biol. 2006;172:177-87.

83. Lupianez DG, Spielmann M, Mundlos S. Breaking TADs: how alterations of chromatin domains result in disease. Trends Genet. 2016;32:225-37.

84. Bonev B, Cavalli G. Organization and function of the 3D genome. Nat Rev Genet. 2016. https://doi.org/10.1038/nrg.2016.147.

85. Yauy K, Gatinois V, Guignard T, Sati S, Puechberty GJB, et al. Looking for broken TAD boundaries and changes on DNA interactions: clinical guide to $3 \mathrm{D}$ chromatin change analysis in complex chromosomal rearrangements and chromothripsis. Methods Mol Biol. 2018;1769:353-62.

86. Middelkamp S, van Heesch S, Braat AK, de Ligt J, van Iterson M, Simonis M, et al. Molecular dissection of germline chromothripsis in a developmental context using patient-derived iPS cells. Genome Med. 2017. https://doi.org/ 10.1186/s13073-017-0399-z.

87. Cavalli G. Chromosome kissing. Curr Opin Genet Dev. 2007;17:443-50.

88. Miné-Hattab J, Rothstein R. Increased chromosome mobility facilitates homology search during recombination. Nat Cell Bio. 2012;14:510-7.

89. Chiolo I, Minoda A, Colmenares SU, Polyzos A, Costes SV, Karpen GH. Double-strand breaks in heterochromatin move outside of a dynamic HP1a domain to complete recombinational repair. Cell. 2011;144:732-44.

90. Krawczyk PM, Borovski T, Stap J, Cijsouw T, ten Cate R, Medema JP, et al. Chromatin mobility is increased at sites of DNA double-strand breaks. J Cell Sci. 2012;125:2117-33.

91. Harewood L, Schütz F, Boyle S, Perry P, Delorenzi M, Bickmore WA, et al. The effect of translocation-induced nuclear reorganization on gene expression. Genome Res. 2010;20:554-64

92. Dion V, Gasser SM. Chromatin movement in the maintenance of genome stability. Cell. 2013;152:1355-64

93. Magrangeas F, Avet-Loiseau H, Munshi NC, Minvielle S. Chromothripsis identifies a rare and aggressive entity among newly diagnosed multiple myeloma patients. Blood. 2011;118:675-8.

94. Molenaar JJ, Koster J, Zwijnenburg DA, van Sluis P, Valentijn $\sqcup$, van der Ploeg I, et al. Sequencing of neuroblastoma identifies chromothripsis and defects in neuritogenesis genes. Nature. 2012;483:589-93.
95. Nagel S, Meyer C, Quentmeier H, Kaufmann M, Drexler HG, MacLeod RA. Chromothripsis in Hodgkin lymphoma. Genes Chromosomes Cancer. 2013; 52(8):741-7.

96. Hirsch D, Kemmerling R, Davis S, Camps J, Meltzer PS, Ried T, et al. Chromothripsis and focal copy number alterations determine poor outcome in malignant melanoma. Cancer Res. 2013;73:1454-60.

97. McEvoy J, Nagahawatte P, Finkelstein D, Richards-Yutz J, Valentine M, Ma J, et al. RB1 gene inactivation by chromothripsis in human retinoblastoma. Oncotarget. 2014;30:438-50.

98. Notta F, Chan-Seng-Yue M, Lemire M, Li Y, Wilson GW, Connor AA, et al. A renewed model of pancreatic cancer evolution based on genomic rearrangement patterns. Nature. 2016;538(7625):378-82.

99. Ratnaparkhe M, Hlevnjak M, Kolb T, Jauch A, Maass KK, Devens F, et al. Genomic profiling of acute lymphblastic leukemia in ataxia telangiectasia patients reveals tight link between ATM mutations and chromothripsis. Leukemia. 2017;31(10):2048-56

100. Zack TI, Schumacher SE, Carter SL, Cherniack AD, Saksena G, Tabak B, et al. Pancancer patterns of somatic copy-number alteration. Nat Genet. 2013;45:1134-40.

101. Kim TM, Luquette L, Park RW, Johnson MD, Park PJ. Functional genomic analysis of chromosomal aberrations in a compendium of 8000 cancer genomes. Genome Res. 2013;23:217-27.

102. Cai H, Kumar N, Bagheri HC, von Mering C, Robinson MD, Baudis M. Chromothripsis-like patterns are recurring but heterogeneously distributed features in a survey of 22,347 cancer genome screens. BMC Genomics. 2014 https://doi.org/10.1186/1471-2164-15-82.

103. Malhotra A, Lindberg M, Faust GG, Leibowitz ML, Clark RA, Layer RM, et al. Breakpoint profiling of 64 cancer genomes reveals numerous complex rearrangements spawned by homology-independent mechanisms. Genome Res. 2013;23(5):762-76.

104. Forment JV, Kaidi A, Jackson SP. Chromothripsis and cancer: causes and consequences of chromosome shattering. Nat Rev Cancer. 2012;12:663-70.

105. Kloosterman WP, Koster J, Molenaar JJ. Prevalence and clinical implications of chromothrispsis in cancer genomes. Curr Opin Oncol. 2014;26:64-72.

106. Luijten MNH, Lee JXT, Crasta KC. Mutational game changer: chromothripsis and its emerging relevance to cancer. Mutat Res. 2018;777:29-51.

107. Ly P, Cleveland DW. Rebuilding chromosomes after catastrophe: emerging mechanisms of chromothripsis. Trends Cell Biol. 2017;27(12):917-30.

108. Garsed Dale W, Marshall Owen J, Corbin Vincent DA, Hsu A, Di Stefano I, Schröder J, et al. The architecture and evolution of cancer neochromosomes. Cancer Cell. 2014;26:653-67.

109. Papenfuss AT, Thomas DM. The life history of neochromosomes revealed. Mol Cell Oncol. 2015. https://doi.org/10.1080/23723556.2014.1000698.

110. Mehine M, Kaasinen E, Mäkinen N, Katainen R, Kämpjärvi K, Pitkänen E, et al. Characterization of uterine leiomyomas by whole-genomesequencing. $\mathrm{N}$ Engl J Med. 2013;369:43-53.

111. Fontana MC, Marconi G, Feenstra JDM, Fonzi E, Papayannidis C, Ghelli Luserna di Rora A, et al. Chromothripsis inacute myeloid leukemia: biological features and impact on survival. Leukemia. 2018; 32(7):1609-20.

112. Willis NA, Rass E, Scully R. Deciphering the code of the cancer genome: mechanisms of chromosome rearrangement. Trend Cancer. 2015;1(4):217-30.

113. Imle A, Polzer B, Alexander S, Klein CA, Friedl P. Genomic instability of micronucleated cells revealed by single-cell comparative genomic hybridization. Cytometry A. 2009;75(7):562-8.

114. Goldschmidt R. The material basis of evolution. UK: Yale University press, New Haven and London; 1940.

115. Eldredge N, Gould SJ. Punctuated equilibria: an alternative to phyletic gradualism. In: Schopf TJM, editor. Models in Paleobiology. Freeman Cooper. USA: San Francisco; 1972. p. 82-115.

116. Reiseberg LH, Archer MA, Wayne RK. Trangressive segregation, adaptation and speciation. Heredity. 1999;83:363-72.

117. Britton-Davidian J, Catalan J, Ramalhinho M, Ganem G, Auffray JC, Capela R, et al. Rapid chromosomal evolution in island mice. Nature. 2000:403:158

118. Carbone L, Harris RA, Gnerre S, Veeramah KR, Lorente-Galdos B, Huddleston J, et al. Gibbon genome and the fast karyotype evolution of small apes. Nature. 2014;513(7517):195-201.

119. Meyer TJ, Held U, Nevonen KA, Klawitter S, Pirzer T, Carbone L, et al. The flow of the gibbon LAVA element facilitated by the LINE-1 retrotransposition machinery. Genome Biol Evol. 2016;8:3209-25.

120. Deakin JE. Chromosome evolution in marsupials. Genes. 2018. https://doi. org/10.3390/genes9020072. 
121. Crombach A, Hogeweg P. Chromosome rearrangements and the evolution of genome structuring and adaptability. Mol Biol Evol. 2007;24(5):1130-9.

122. Kass EM, Moynahan ME, Jasin M. When genome maintenance goes badly awry. Mol Cell. 2016;62(5):777-87.

123. Fukami M, Shima H, Suzuki E, Ogata T, Matsubara K, Kamimaki T. Catastrophic cellular events leading to complex chromosoma rearrangements in the germline. Clin Genet. 2017;91:653-60.

124. Pellestor F, Gatinois V, Puechberty J, Geneviève D, Lefort G. Chromothripsis: potential origin in gametogenesis and preimplantation cell divisions. A review. Fertil Steril. 2014;102:1785-96.

125. Tan EH, Henry IM, Ravi M, Bradnam KR, Mandakova T, Marimuthu MPA, et al. Catastrop hic chromosomal restructuring during genome elimination in plants. eLife. 2015. https://doi.org/10.7554/eLife.06516.

126. Itani OA, Flibotte S, Dumas K, Moerman DG, Hu PJ. Chromoanasynthetic genomic rearrangement identified in a N-ethyl-N-Nitrosourea (ENU) mutageneis screen in Caenorhabditis elegans. G3 (Bethesda). 2015;6(2):351-6.

127. Anand RP, Tsaponina O, Greenwell PW, Lee CS, Du W, Petes TD, et al. Chromosome rearrangements via template switching between diverged repeated sequences. Genes Dev. 2014;28(21):2394-406.

128. Liu G, Stevens JB, Horne SD, Abdallah BY, Ye KJ, Bremer SW, et al. Genome chaos. Survival strategy during crisis. Cell Cycle. 2014;13:1-10.

129. Ye CJ, Liu G, Heng HH. Experimental induction of genome chaos. Methods Mol Biol. 2018;1769:337-52.

Ready to submit your research? Choose BMC and benefit from:

- fast, convenient online submission

- thorough peer review by experienced researchers in your field

- rapid publication on acceptance

- support for research data, including large and complex data types

- gold Open Access which fosters wider collaboration and increased citations

- maximum visibility for your research: over $100 \mathrm{M}$ website views per year

At $\mathrm{BMC}$, research is always in progress.

Learn more biomedcentral.com/submissions 